\title{
Protective effect of Fructus corni polysaccharide on hippocampal tissues and its relevant mechanism in epileptic rats induced by lithium chloride-pilocarpine
}

\author{
XIAOMIN SUN ${ }^{1 *}$, LINGTING KONG ${ }^{2 *}$ and LI ZHOU ${ }^{1}$ \\ ${ }^{1}$ Department of Neurology, Weifang People's Hospital, Weifang, Shandong 261041; \\ ${ }^{2}$ Department of Pharmacy, People's Hospital of Gaotang County, Liaocheng, Shandong 252800, P.R. China
}

Received January 26, 2018; Accepted March 16, 2018

DOI: $10.3892 /$ etm.2018.6142

\begin{abstract}
The aim of the present study was to investigate the potential effect of Fructus corni polysaccharide (PFC) on the hippocampus tissues in epileptic rats induced by lithium chloride-pilocarpine, and to explore the underlying mechanism. The epileptic rat models were established using lithium chloride-pilocarpine treatment. According to the dosage of PFC, the rat models were divided into three groups: The low-dose (100 mg/kg/day), middle-dose (200 mg/kg/day) and high-dose (300 mg/kg/day) groups. The intervention for rat models lasted for 24 days. Subsequently, the production levels of reactive oxygen species (ROS) and malondialdehyde (MDA), the activity of superoxide dismutase (SOD), the mitochondrial membrane potential and the expressions of mitogen-activated protein kinase [P-38, Janus kinase (JNK) and extracellular signal-regulated kinase $1 / 2$ ], cytochrome-C and caspase- 3 in hippocampal tissues were detected. In addition, the structure of the CA-1 region of the hippocampus was also observed. Compared with the control group, the production levels of ROS were increased and the mitochondrial membrane potential was decreased in the hippocampus tissues of rats in the model group. In addition, in the model group, it was observed that MDA content was increased, SOD activity was decreased, and the expressions of phosphorylated (p)-p38, p-JNK, cytochrome-c and caspase-3 were increased, compared with the control group. Furthermore, those abnormal variations of the indicators were reversed by the intervention of PFC. These findings suggest that PFC can ameliorate the secondary damage to the hippocampi of epileptic rats, and that
\end{abstract}

Correspondence to: $\mathrm{Dr} \mathrm{Li}$ Zhou, Department of Neurology, Weifang People's Hospital, 151 Guangwen Street, Kuiwen, Weifang, Shandong 261041, P.R. China

E-mail: h0i422@163.com

*Contributed equally

Key words: Fructus corni, mitogen-activated protein kinase, reactive oxygen species, apoptosis, epilepsy the anti-oxidation and -apoptosis effects of PFC may be associated with the mechanism that provides a protective effect for hippocampal tissues.

\section{Introduction}

Epilepsy is a common neurological syndrome, the recurrence of which is apt to induce damages to brain tissues in varying degrees, including cognitive dysfunction or damage to the structure and functions of mitochondria $(1,2)$. At present, anti-epilepsy drugs in clinical practice are only designed for certain specific targets, and long-term administration may make patients more susceptible to a series of serious adverse reactions, including hematopoietic system damage, Stevens-Johnson syndrome, severe hepatic dysfunction (3) or aggravate cognitive dysfunction (3). Therefore, identifying a novel drug that can effectively improve cognitive functions without side-effects or toxicity has been considered as a priority.

Oxidative stress refers to the oxidative damage caused by reactive oxygen species (ROS). The excessive generation of free radicals or the dysfunction of in vivo anti-oxidation systems result in imbalance in the metabolism of oxygen radicals, which may damages tissues and thus the body as a whole (4). ROS may also act on the mitochondria (5), leading to changes in permeability of the mitochondrial membrane, which can contribute to the apoptosis of neurons and make patients more susceptible to the onset of epilepsy $(6,7)$. Thus, cell apoptosis has an important role in the pathogenesis of epilepsy.

Mitogen-activated protein kinase (MAPK) pathways are important transmembrane signal transduction pathways, primarily including three major MAPK cascade reactions: p38, extracellular signal-regulated kinase (ERK) $1 / 2$ and c-Jun $\mathrm{N}$-terminal kinase (JNK) (8). These cascade reactions are not only associated with cell growth, proliferation and apoptosis, but also have key roles in these processes. p38, ERK1/2 and JNK are all sensitive to the stimulation of $\operatorname{ROS}(9,10)$.

Fructus corni polysaccharide (PFC), obtained from the Traditional Chinese Medicine Fructus corni, is one of the most common Traditional Chinese Medicines (11). Previous pharmacological studies have suggested that PFC has a 
wide range of biological activities, including: Antioxidative, anti-inflammatory, immuno-modulatory, antidiabetic, and hypoglycemic $(12,13)$. Thus, the antioxidant activity of PFC may have potential benefits in protecting against epilepsy.

In the present study, an epileptic rat model was established through the induction of lithium chloride-pilocarpine to investigate the potential effect of PFC on mitochondrial damage in hippocampal tissues and the potential underlying mechanism, thereby identifying novel pathways and theoretical evidence for the clinical treatment of epilepsy.

\section{Materials and methods}

Reagents. PFC (95\%) was provided by Ningbo Dekang Biological Products Co., Ltd. (Ningbo, China). Lithium chloride and pilocarpine (Sigma-Aldrich; Merck KGaA, Darmstadt, Germany), were used to establish the epileptic rat model. Malondialdehyde (MDA; cat. no. JC201712) content and superoxide dismutase (SOD; cat. no. JC201722) activity in hippocampal tissue were detected with ELISA detection kits provided by Nanjing Jiancheng Bioengineering Institute (Nanjing, China). ROS level was detected by flow cytometry (BD FACSAria $^{\mathrm{TM}}$ II; BD Biosciences, San Jose, CA, USA) using the oxidation sensitive fluorescent dye, 2',7'-dichlorodihydrofluorescein diacetate (DCFH-DA; CAS:D6883; Sigma-Aldrich; Merck KGaA).

Study design. Rats were randomly assigned into the following five groups: I: Control group $(n=15)$, which was administered saline (vehicle) via gavage for 44 days (6 ml/kg/day); II: Lithium-pilocarpine (LP) group $(\mathrm{n}=15)$, which was administered saline ( $6 \mathrm{ml} / \mathrm{kg} / \mathrm{day})$ for 24 days followed by lithium-pilocarpine treatment for 20 days (model establishment); III, IV and V ( $\mathrm{n}=15$ per group): $\mathrm{LP}+\mathrm{PFC}$ groups that were administered PFC (100, 200 or $300 \mathrm{mg} / \mathrm{kg} /$ day $)$ for 24 days followed by lithium-pilocarpine treatment for 20 days. The mortality and seizure stages of the rats were recorded throughout.

Model establishment and screening. A total of 120 male Sprague Dawley rats (age, 6-8 weeks; weight, 180-220 g) were used in the present study; they were purchased from the Shandong Province Animal Research Center (Shandong, China). The rats had free access to food and water and were housed in a temperature controlled room $\left(21 \pm 2^{\circ} \mathrm{C}\right)$ in a $12 \mathrm{~h}$ light/dark cycle. Rats received an intraperitoneal injection of $3 \mathrm{mmol} / \mathrm{kg}$ lithium chloride $(127 \mathrm{mg} / \mathrm{kg})$, and after $18-24 \mathrm{~h}$, atropine sulfate $(1 \mathrm{mg} / \mathrm{kg}$; Sigma-Aldrich; Merck KGaA, Darmstadt, Germany) was administered to rats to antagonize the peripheral cholinergic effect of pilocarpine, which was initially administered following another $30 \mathrm{~min}(20 \mathrm{mg} / \mathrm{kg})$. The behavior of rats was observed and graded based on the Racine classification system (14). After $30 \mathrm{~min}$ if no epileptic seizures were observed, pilocarpine $(10 \mathrm{mg} / \mathrm{kg})$ was administered to the rats without the onset of status epilepticus (SE) every 30 min until the onset of SE was successfully induced. Rats that failed in the model establishment were excluded. For rats with SE lasting for $1 \mathrm{~h}$, intraperitoneal administration of $10 \%$ chloral hydrate $(350 \mathrm{mg} / \mathrm{kg}$ ) (Sigma-Aldrich; Merck $\mathrm{KGaA}$ ) was performed to terminate the seizure, and additional doses were administered if a poor effect was observed $30 \mathrm{~min}$ following the previous injection. Surviving rats would enter the quiet phase $24 \mathrm{~h}$ after modeling as previously described (15), the toxic stage consisted of the initial 2-4 days. Rats would suffer spontaneous epilepsy 7-20 days after modeling. A total of 60 rats with seizures above Racine grade IV were considered as successful spontaneous epileptic models, and those that failed to meet the criterion were excluded. Animal protocols were approved by the Animal Ethics Committee of Weifang People's Hospital Animal Center (Weifang, China).

Behavioral assessment. Behavioral assessment was performed during the model establishment and every day following successful modeling in accordance with Racine's level 6 evaluation standards (14), and scored as follows: Stage 0, no response; stage 1, vibrissae twitching and hyperactivity; stage 2 , clonus, head nodding and myoclonic jerks of the head; stage 3 , unilateral forelimb clonus; stage 4 , rearing with bilateral forelimb clonus; and stage 5 , generalized tonic-clonic seizure with loss of writing reflex.

Detection of MDA content and SOD activity. Rats were sacrificed after the 44 day treatment period and the hippocampus was immediately removed and homogenized in ice-cold normal saline. Following cooling, the samples were centrifuged at 2,500 $\mathrm{xg}$ at $4^{\circ} \mathrm{C}$ for $20 \mathrm{~min}$. Supernatants were taken to determine the levels of MDA and SOD using ELISA kits according to the manufacturer's protocols.

Detection of mitochondrial ROS production. As described previously (16), dichlorohydrofluorescein diacetate (DCFH-DA) was used for accurate measurement of production of mitochondrial ROS. Rats were sacrificed and hippocampus tissues were rapidly obtained and homogenized. DCFH-DA fluorescence was used for measurement at $488 \mathrm{~nm}$ excitation and $530 \mathrm{~nm}$ emission.

Detection of mitochondrial membrane potential. Rhodamine 123 (Rh-123) staining was used to determine the mitochondrial membrane potential in rats according to a previous study (17). Mitochondria were then pelleted by centrifugation at $8,600 \mathrm{xg}$ at $4^{\circ} \mathrm{C}$ for $15 \mathrm{~min}$ and fluorescence of the supernatant was measured at $503 \mathrm{~nm}$ excitation and $527 \mathrm{~nm}$ emission using a spectrophotometer.

Nissl staining. Following sacrifice, rat brains were immediately harvested, fixated with $4 \%$ paraformaldehyde at $20^{\circ} \mathrm{C}$ for $24 \mathrm{~h}$, and then embedded in paraffin. Subsequently, the paraffin-embedded brains were cut into $10-\mu \mathrm{m}$ thick sections for Nissl staining with toluidine blue to evaluated general neuronal morphology. After being flushed with distilled water, the sections were placed in a $37^{\circ} \mathrm{C}$ water bath and incubated for 10 min with $0.5 \%$ thionine. The staining results were observed under a light microscope (magnification, $\mathrm{x} 400$ ). The neuronal density in the CA1 region of the hippocampus was calculated as previously described $(18,19)$.

Western blot analysis. Hippocampi were collected and homogenized in ice using homogenization buffer, then centrifuged at $13,200 \mathrm{x}$ g for $15 \mathrm{~min}$ at $4^{\circ} \mathrm{C}$. Supernatants were collected, and 
A
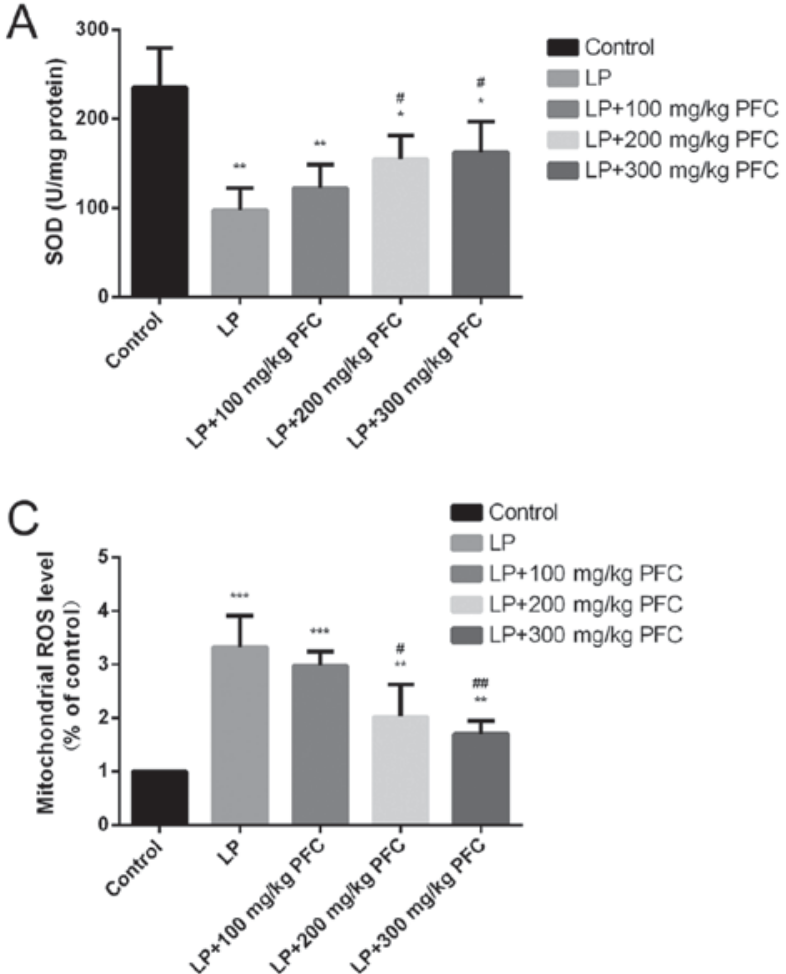

B

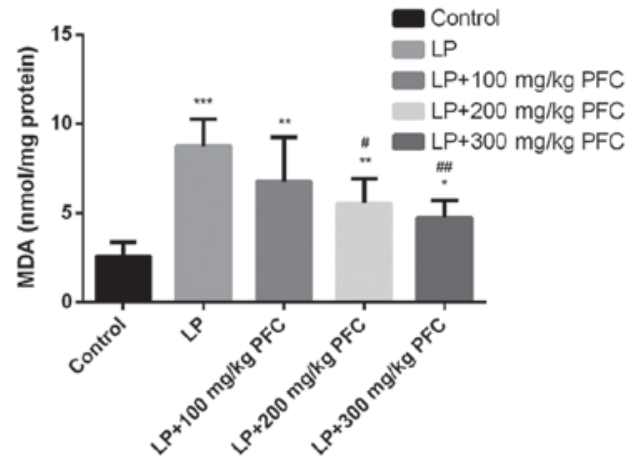

$\mathrm{D}$

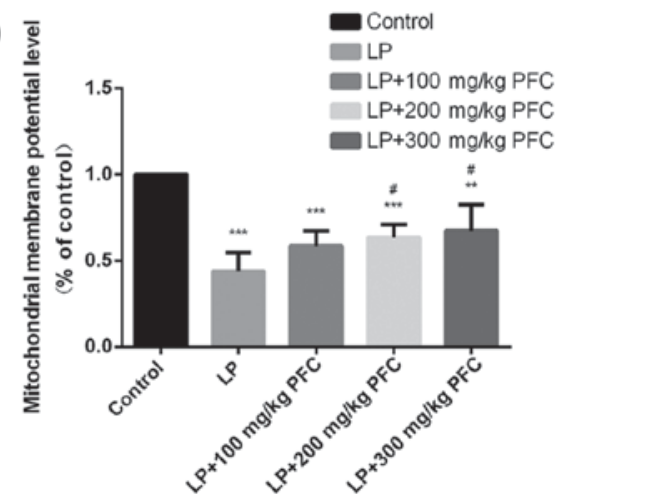

Figure 1. Effects of PFC on hippocampal MDA content, SOD activity, mitochondrial ROS generation and the mitochondrial membrane potential. (A) Effect of PFC on SOD activity. Compared with the control group, SOD activity was significantly decreased in the LP group. Compared with the LP injury group, SOD activity was increased in the LP+PFC groups. (B) Effect of PFC on MDA content. Compared with control group, MDA content was significantly increased in LP group. Compared with the LP injury group, MDA content was significantly decreased in the LP+PFC groups. (C) Effect of PFC on mitochondrial ROS production. Compared with the control group, mitochondrial ROS production was significantly increased in the LP group. Compared with the LP injury group, the mitochondrial ROS production was decreased in the LP+PFC groups. (D) Effect of PFC on mitochondrial membrane potential. Compared with the control group, the mitochondrial membrane potential was significantly decreased in the LP group. Compared with the LP group, the mitochondrial membrane potential was increased in the LP+PFC groups. ${ }^{*} \mathrm{P}<0.05,{ }^{* *} \mathrm{P}<0.01,{ }^{* * * *} \mathrm{P}<0.001$ vs. control group; ${ }^{\#} \mathrm{P}<0.05,{ }^{\# \#} \mathrm{P}<0.01$ vs. LP group. Data are presented as the mean + standard deviation ( $\mathrm{n}=5$ ). PFC, Fructus corni polysaccharide; SOD, superoxide dismutase; MDA, malondialdehyde; ROS, reactive oxygen species; LP, lithium-pilocarpine.

a bicinchoninic acid protein assay kit was used to calculate the protein concentrations. Cytoplasmic and mitochondrial proteins were each isolated via differential centrifugation with a mitochondrial fractionation kit (Nanjing KeyGen Biotech Co., Ltd., Nanjing, China) according to the manufacturer's instructions. Protein samples $(50 \mu \mathrm{g})$ were subjected to $10 \%$ SDS-PAGE. Following electrophoresis, proteins were transferred to polyvinylidene difluoride membranes and blocked with 5\% (vol/vol) non-fat dry milk in Tris-buffered saline with Tween-20 (TBST; $10 \mathrm{mM}$ Tris- $\mathrm{HCl}, 150 \mathrm{mM} \mathrm{NaCl}$ and $0.1 \%$ Tween-20; $\mathrm{pH} 7.6$ ) at room temperature for $2 \mathrm{~h}$, followed by incubation overnight with 1:1,000 dilutions of cleaved caspase-3 (cat. no. 9661S), p38 MAPK (cat. no. 8690S), phosphorylated (p-)p38 MAPK (cat. no. 4511S), ERK (cat. no. 4695S), p-ERK (cat. no. 4370S), JNK (cat. no. 9252S), p-JNK (cat. no. 9255S) (all Cell Signaling Technology, Inc., Danvers, MA, USA) and cytochrome C (cat. no. sc-13561; Santa Cruz Biotechnology, Inc., Dallas, TX, USA) primary antibodies. $\beta$-actin (dilution, 1:2,000; cat. no. sc-517582; Santa Cruz Biotechnology, Inc.) served as the internal control. The membrane was washed in TBST 3 times for 5 min each. The membrane was subsequently incubated with horseradish peroxidase-conjugated secondary antibody (cat. no. 5571S; 1:1,000; Cell Signaling Technology, Inc.) at room temperature for an additional $2 \mathrm{~h}$, and washed an additional 3 times in TBST. The membrane was subsequently incubated with enhanced chemiluminescence substrate solution at $20^{\circ} \mathrm{C}$ for $5 \mathrm{~min}$ according to the manufacturer's protocol (cat. no. orb90504; Biorbyt, Cambridge, UK) and visualized using autoradiography film. The imaging program Quantity One 4.6.2 (Bio-Rad Laboratories, Inc., Hercules, CA, USA) was used for quantification.

Statistical analysis. Data are presented as the mean + standard deviation. The statistical analyses were performed using GraphPad Prism 5.01 (GraphPad Software, Inc., La Jolla, CA, USA) and PASW 18.0 (SPSS, Inc., Chicago, IL, USA). One-way analysis of variance was used to compare differences among multiple groups, followed by the least significant difference post hoc test. Three independent repeats were performed for each experiment. $\mathrm{P}<0.05$ was considered to indicate a statistically significant difference.

\section{Results}

Effects of PFC on SOD activity and MDA content. Compared with the control group, SOD activities significantly decreased $(\mathrm{P}<0.01)$ and the MDA content was significantly increased $(\mathrm{P}<0.001)$ in the LP group. Gavage with $200 \mathrm{mg} / \mathrm{kg}$ $\mathrm{PFC}(\mathrm{P}<0.05)$ and $300 \mathrm{mg} \mathrm{PFC}(\mathrm{P}<0.01)$ significantly reduced the content of MDA and upregulated the activity of SOD compared with LP group. These findings suggest that PFC treatment may ameliorate oxidative stress (Fig. 1A and B). 

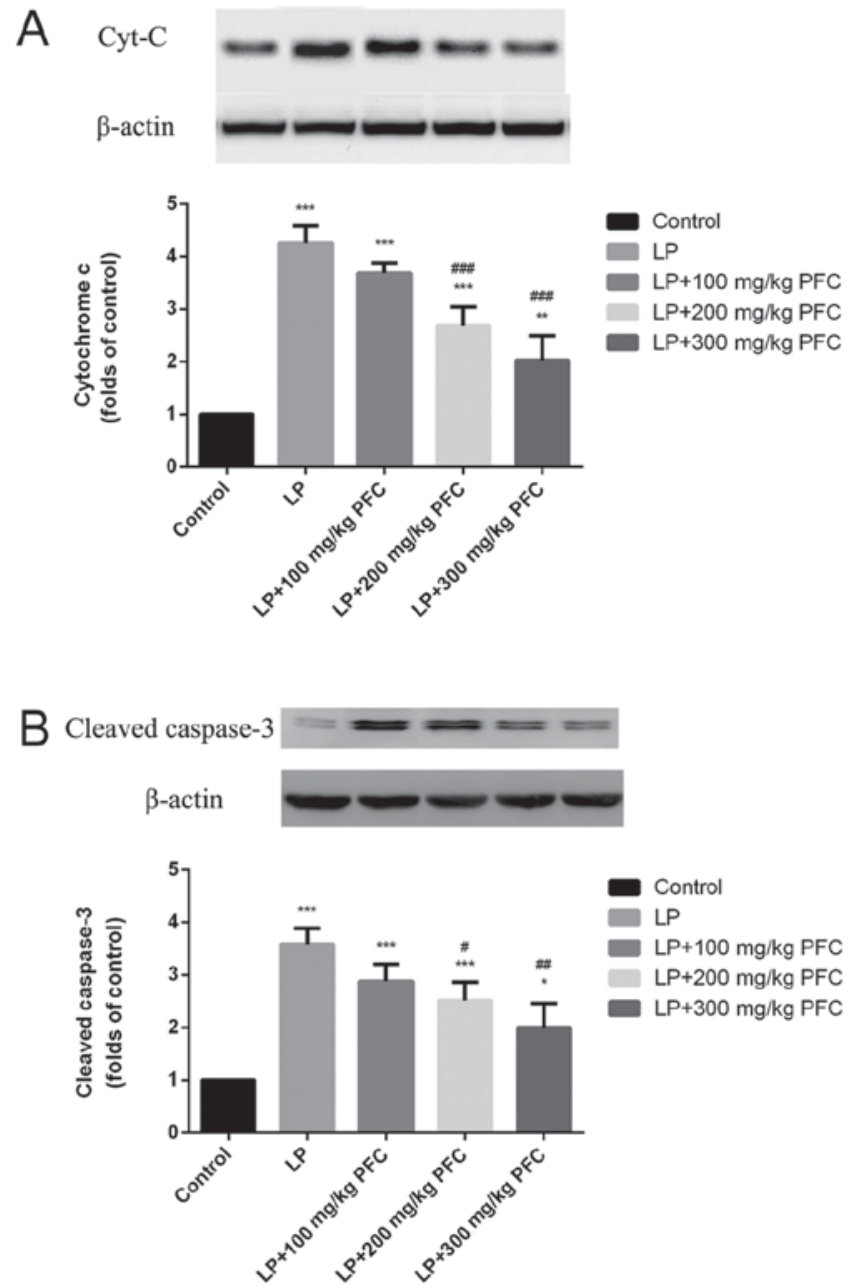

Figure 2. Effects of PFC on the release of Cyt-C and the activation of cleaved caspase-3 in the hippocampus. PFC administration (A) decreases Cyt-C release and (B) prevents cleaved caspase- 3 activation. Cyt- $\mathrm{C}$ release and cleaved caspase-3 activation was increased in the LP groups compared with the control group. Treatment with PFC decreased Cyt-C and cleaved caspase-3 levels. ${ }^{*} \mathrm{P}<0.05,{ }^{* *} \mathrm{P}<0.01,{ }^{* * *} \mathrm{P}<0.001$ vs. control group; ${ }^{*} \mathrm{P}<0.05$, ${ }^{\# \#} \mathrm{P}<0.01,{ }^{\# \#} \mathrm{P}<0.001$ vs. LP group. Data are presented as the mean + standard deviation $(n=5)$. PFC, Fructus corni polysaccharide; Cyt-C, cytochrome C; LP, lithium-pilocarpine.

Effects of PFC on mitochondrial ROS production induced by epilepsy. The present results demonstrated that the level of mitochondrial ROS formation was significantly increased in LP group compared with control $(\mathrm{P}<0.001)$. However, treatment with $200 \mathrm{mg} / \mathrm{kg}$ PFC $(\mathrm{P}<0.05)$ and $300 \mathrm{mg}$ PFC $(\mathrm{P}<0.01)$ significantly decreased the levels of mitochondrial ROS production, compared with the LP group (Fig. 1C).

Effect of PFC on mitochondrial membrane potential modification induced by epilepsy. In the LP group the mitochondrial membrane potential was significantly reduced compared with the control group $(\mathrm{P}<0.001)$. Treatment with PFC $(200$, $300 \mathrm{mg} / \mathrm{kg}$ ) significantly ameliorated this decline in mitochondrial membrane potential $(\mathrm{P}<0.05$; Fig. 1D).

Effect of PFC on cytochrome $C$ and activation of cleaved-caspase-3 following epilepsy. Via western blotting, the release of cytochrome $\mathrm{C}$ was demonstrated to significantly increase in the LP group compared with control $(\mathrm{P}<0.001$; Fig. 2A). Cleaved caspase-3 is a characteristic sign of apoptosis (20). It was also demonstrated that cleaved caspase-3 production significantly increased in LP groups $(\mathrm{P}<0.001$; Fig. 2B). Treatment with $200 \mathrm{mg} / \mathrm{kg}$ PFC (P<0.001) and $300 \mathrm{mg}$ PFC $(\mathrm{P}<0.001)$ significantly ameliorated this cytochrome $\mathrm{C}$ release. Treatment with $200 \mathrm{mg} / \mathrm{kg}$ PFC $(\mathrm{P}<0.05)$ and $300 \mathrm{mg}$ PFC $(\mathrm{P}<0.01)$ significantly reduced cleaved-caspase-3 activation.

Effect of PFC on MAPK phosphorylation in epileptic rats. The MAPK family comprises the following 3 primary subfamilies: ERK1/2, JNK, and p38. The phosphorylation of p38 and JNK levels were significantly increased in LP groups compared with control $(\mathrm{P}<0.001$; Fig. $3 \mathrm{~A}$ and $\mathrm{B})$. However, induction of epilepsy had no significant effect in inducing the phosphorylation of ERK (Fig. 3C). The administration of $200 \mathrm{mg} / \mathrm{kg} \mathrm{PFC}$ $(\mathrm{P}<0.05)$ and $300 \mathrm{mg} \mathrm{PFC}(\mathrm{P}<0.01)$ significantly ameliorated the increases in p38 and JNK phosphorylation, compared with the LP group.

PFC retrieved hippocampal neurons in CAl area. As presented in Fig. 4, neurons in the control group (Fig. 4A) appeared as light blue, regularly shaped cell bodies with palely stained nuclei in the Nissl-stained sections. However, neurons in the LP group (Fig. 4B) exhibited blurred cell membranes and pyknotic nuclei. Furthermore, the mean density of intact surviving neurons was significantly lower compared with control ( $\mathrm{P}<0.001$; Fig. 4F). These morphological abnormalities were alleviated in PFC groups (Fig. 4C-F), which indicates that PFC treatment has a beneficial influence on damaged neurons in the CA1 region.

\section{Discussion}

Epilepsy is frequently characterized by complicated pathogenesis. It has been observed that oxidative stress and apoptosis have a vital role in the pathological process of epilepsy in previous studies $(21,22)$, and that a causal relation exists between them. Under normal circumstances, the body can physiologically generate a small amount of oxygen free radicals (4). The ROS generated in the electron transfer of the respiratory chain in mitochondria can be eradicated by the endogenous anti-oxidation system in cells to sustain the balance in the body (23). The anti-oxidation system in the brain tissues, which is relatively inferior to that in other tissues or organs, can be affected by various factors, such as ischemia or hypoxia (4). The brain is susceptible to the imbalance between the oxidation system and anti-oxidation system in cells (24). Excessive ROS in the body may give rise to the massive accumulation of free radicals, resulting in a further marked increase of ROS (4). Meanwhile, ROS may act on the unsaturated fatty acids found on cellular membrane and mitochondrial membrane, inducing lipid peroxidation and cell apoptosis (25). In the present study, it was demonstrated that ROS level the MDA content in hippocampal tissues were significantly increased, and the activity of SOD (an important indicator of endogenous anti-oxidation system in cells) was significantly decreased in the LP group compared with controls. This confirmed that the epileptic seizure induced by 

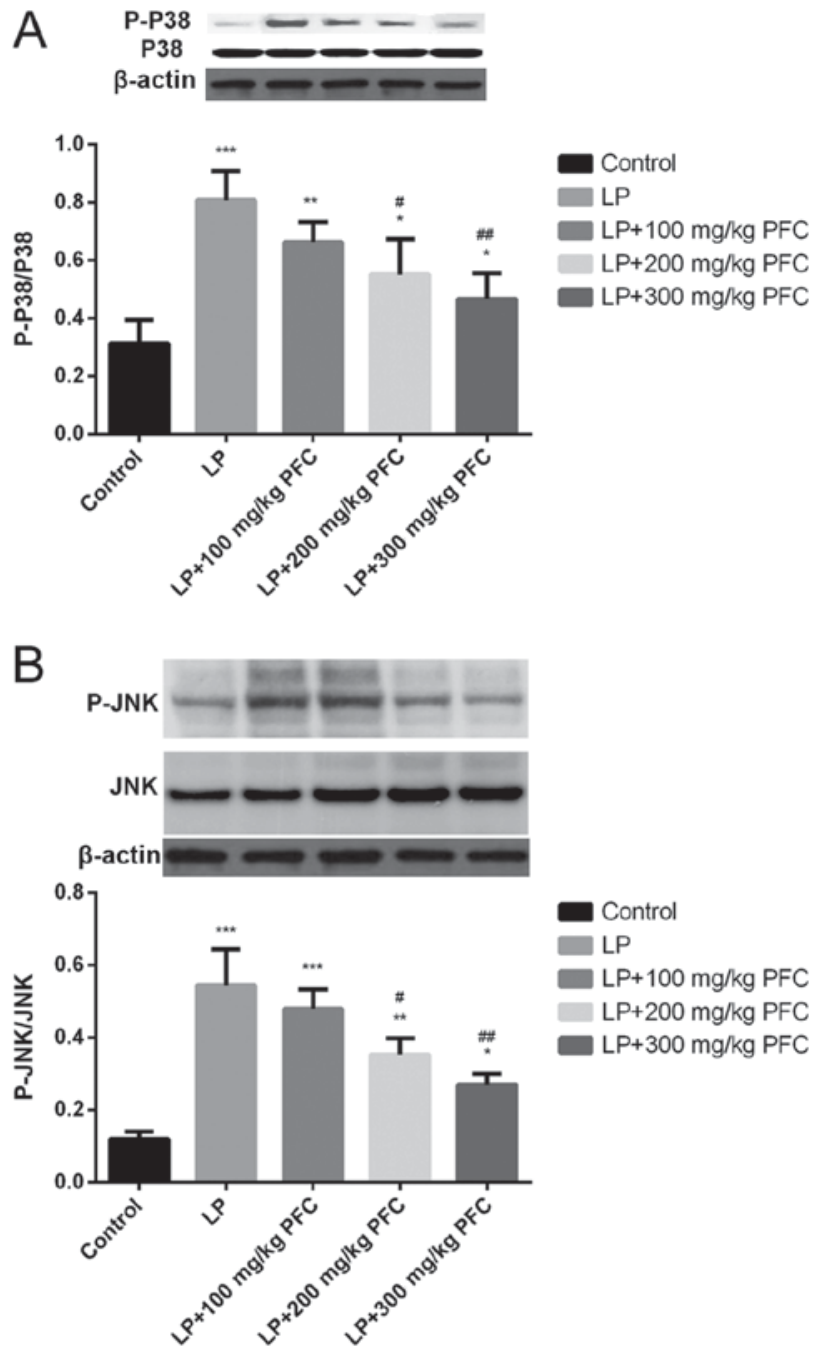

$\mathrm{LP}+300 \mathrm{mg} / \mathrm{kg}$ PFC

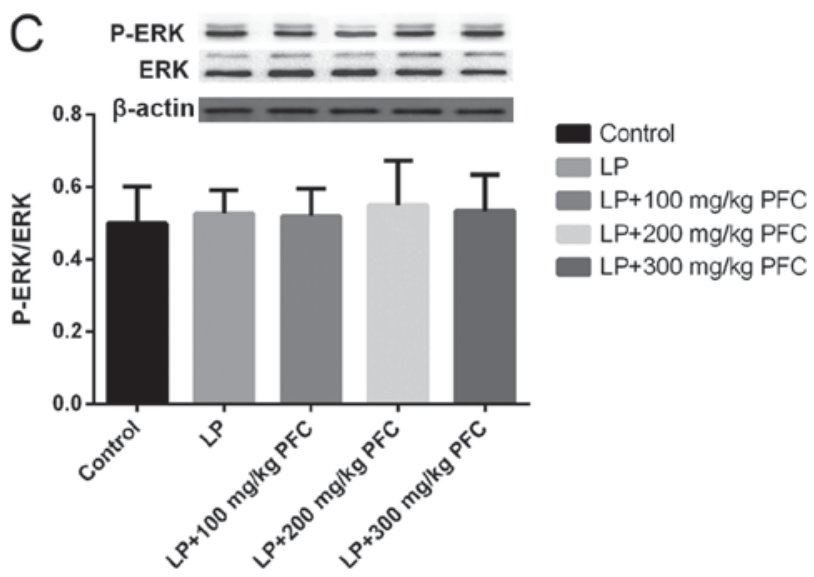

Figure 3. Effects of PFC on the phosphorylation of kinase in mitogen-activated protein kinase signal pathways, namely P-38, JNK and ERK. Representative western blotting and densitometry data for the levels of (A) p-p38/p38, (B) p-JNK/JNK and (C) p-ERK/ERK in each group. PFC administration prevented the increases in p38 and JNK phosphorylation. Induction of epilepsy did not induce the phosphorylation of ERK. ${ }^{*} \mathrm{P}<0.05$, ${ }^{* *} \mathrm{P}<0.01,{ }^{* * *} \mathrm{P}<0.001$ vs. control group; ${ }^{\#} \mathrm{P}<0.05,{ }^{\# \#} \mathrm{P}<0.01$ vs. LP group. Data are presented as the mean + standard deviation $(n=5)$. PFC, Fructus corni polysaccharide; JNK, c-Jun N-terminal kinase; ERK, extracellular signal-regulated kinase; $p$, phosphorylated; LP, lithium-pilocarpine.

lithium chloride-pilocarpine induced stress and damaged the hippocampal tissues.
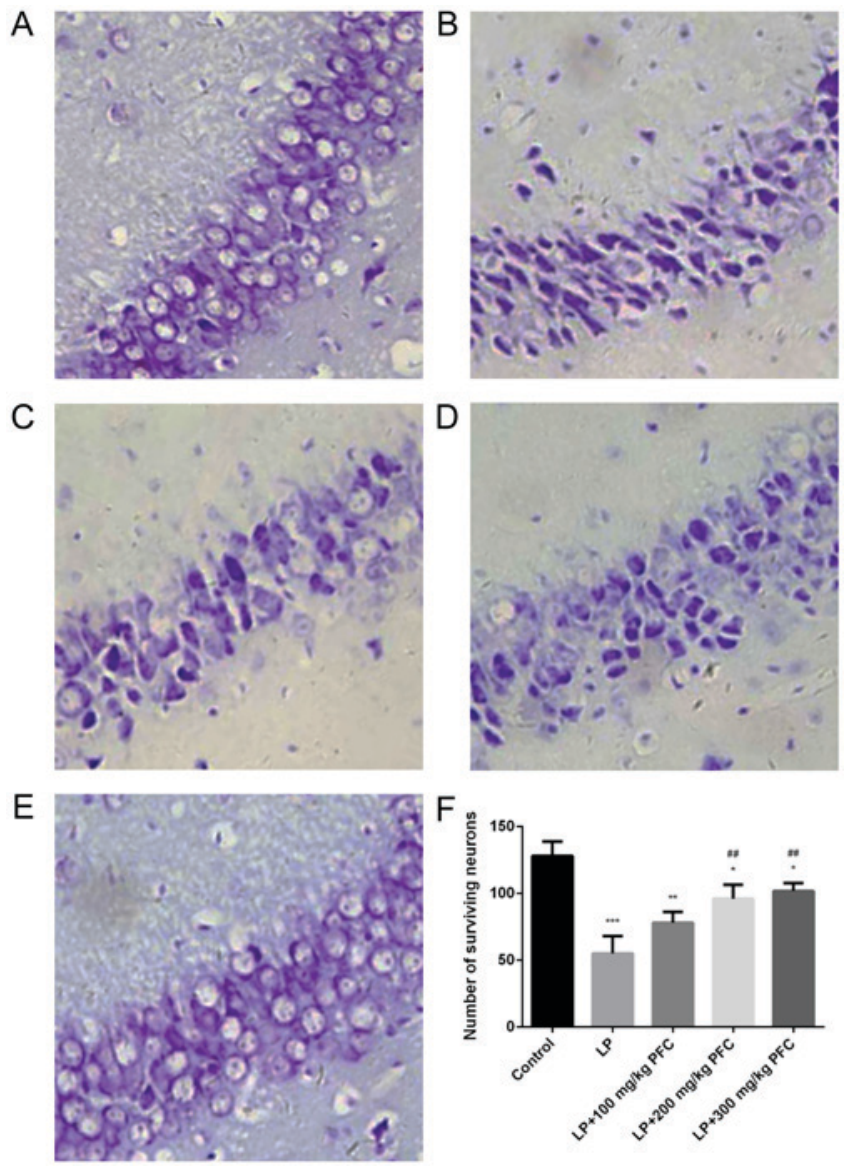

Figure 4. PFC rescues CA1 pyramidal neurons from seizure-induced damage as revealed by Nissl staining. (A) control group, (B) LP group, (C) LP + $100 \mathrm{mg} / \mathrm{kg}$ PFC group, (D) LP + $200 \mathrm{mg} / \mathrm{kg}$ PFC group, (E) LP + $300 \mathrm{mg} / \mathrm{kg}$ PFC group (magnification, $\mathrm{x} 400$ ). (F) Compared with the LP group, treatment with PFC significantly ameliorated the number of surviving neurons. ${ }^{*} \mathrm{P}<0.05,{ }^{* *} \mathrm{P}<0.01,{ }^{* * *} \mathrm{P}<0.001$ vs. control group; ${ }^{\# \#} \mathrm{P}<0.01$ vs. LP group. Data are presented as the mean + standard deviation $(n=5)$. PFC, Fructus corni polysaccharide; LP, lithium-pilocarpine.

Mitochondria not only serve as the main source of endogenous ROS, but also the action target of ROS $(26,27)$. Mitochondrial dysfunction can increase the generation of ROS and aggravate cell apoptosis induced by oxidant (28). Oxidative stress injuries can lead to an imbalance between the oxidative effect and the anti-oxidative effect in the body, thereby massively increasing the generation of ROS, which will cause the mitochondrial permeability transition pore to open (29). As a result, cytochrome-C from the mitochondria will be delivered into the cytoplasm, where caspase-3, the apoptotic executioner, will be activated to induce cell apoptosis $(30,31)$.

ROS, as a kind of signal molecule, can affect the activities of a variety of signal transduction pathways, such as the Janus kinase/signal transducer and activator of transcription pathway, the protein kinase $C$ pathway and the MAPK pathway. Among those pathways, MAPK is a key transmembrane signal transduction pathway (32). In the present study, it was further investigated whether MAPK signal transduction pathway was activated in neural apoptosis induced by ROS. It was observed that the phosphorylation levels of p38 and JNK signal transduction pathways in hippocampus tissues were significantly augmented. Following treatment with PFC, phosphorylation 
levels were significantly decreased, suggesting that the protection mechanism of PFC may be associated with the decreased phosphorylation of kinase in the MAPK signal pathway.

In recent years, neuronal apoptosis has received increasing attention in the field of epilepsy research. It has been argued that the prevention and treatment of neuronal apoptosis following epilepsy has important clinical significance. Following epileptic seizure, neurons in the brain are damaged and lost, of which the hippocampal neurons are lost most significantly $(33,34)$. It has been suggested that neuronal loss occurs in the hippocampal dentate gyrus region, and CA1 and CA3 regions in different degrees in model rats, which may be in the forms of either neuronal necrosis or apoptosis. It has typically been suggested that cell apoptosis is the primary form of brain neuronal death following epilepsy $(35,36)$. The occurrence of apoptosis is regulated by genes, and ROS may cause apoptosis through a variety of ways. The MAPK cascade pathway is an important example $(37,38)$. It has been demonstrated that MAPK phosphorylation can lead to the release of mitochondrial cytochrome $\mathrm{C}$ and then cause the activation of caspase (39). Fructus corni, a kind of Traditional Chinese Medicine, is one of 40 different types of commonly used medicinal materials in China, and is widely used in the traditional and clinical medication. In recent years, progress has been made in the study on PFC $(11,40)$. Pharmacological studies have demonstrated that the PFC is an important component of the bio-active material of Fructus corni (41). Previous studies have demonstrated that PFC exhibits many pharmacological effects, including anti-aging, anti-tumor, anti-oxidation, immuno-enhancement and memory improvement $(42,43)$.

The present study demonstrated that PFC serves as a novel agent for anti-epilepsy treatment. It decreases the alteration in mitochondrial membrane potential, cytochrome $\mathrm{C}$ leakage and activation of cleaved caspase- 3 through reducing the activation of hippocampus ROS and the MAPK cascade pathway following epilepsy, thereby alleviating the apoptosis of neurons and having a neuroprotective effect on epilepsy. In this way, PFC has therapeutic and protective effects on epilepsy, but the specific mechanism of PFC for epileptic seizure remains to be elucidated. The present study was limited to observing the changes in the PFC protein, further investigation is required to study the potential mechanisms by which PFC may have an effect.

\section{Acknowledgements}

Not applicable.

\section{Funding}

Not applicable.

\section{Availability of data and materials}

All data generated or analyzed during the present study are included in this article.

\section{Authors' contributions}

XS, LK and LZ contributed to the study design. XS performed data collection, XS performed data analysis and LK performed data interpretation. XS and LK were responsible for manuscript preparation and LK performed literature search. All authors read and approved the final manuscript.

\section{Ethics approval and consent to participate}

The present study was approved by the Animal Ethics Committee of Weifang People's Hospital Animal Center (Weifang, China).

\section{Consent for publication}

Not applicable.

\section{Competing interests}

The authors declare that they have no competing interests.

\section{References}

1. Chen J, Liu XM, Yue X and Chen SZ: The clinical efficacy and safety of levetiracetam add-on therapy for child refractory epilepsy. Eur Rev Med Pharmacol Sci 20: 2689-2694, 2016.

2. Azakli H, Gurses C, Arikan M, Aydoseli A, Aras Y, Sencer A, Gokyigit A, Bilgic B and Ustek D: Whole mitochondrial DNA variations in hippocampal surgical specimens and blood samples with high-throughput sequencing: A case of mesial temporal lobe epilepsy with hippocampal sclerosis. Gene 529: 190-194, 2013.

3. Mehla J, Reeta KH, Gupta P and Gupta YK: Protective effect of curcumin against seizures and cognitive impairment in a pentylenetetrazole-kindled epileptic rat model. Life Sci 87: 596-603, 2010

4. Valko M, Rhodes CJ, Moncol J, Izakovic M and Mazur M: Free radicals, metals and antioxidants in oxidative stress-induced cancer. Chem Biol Interact 160: 1-40, 2006.

5. Hass DT and Barnstable CJ: Uncoupling protein 2 in the glial response to stress: Implications for neuroprotection. Neural Regen Res 11: 1197-1200, 2016.

6. Baille V, Clarke PG, Brochier G, Dorandeu F, Verna JM, Four E, Lallement G and Carpentier P: Soman-induced convulsions: The neuropathology revisited. Toxicology 215: 1-24, 2005.

7. Sfaello I, Baud O, Arzimanoglou A and Gressens P: Topiramate prevents excitotoxic damage in the newborn rodent brain. Neurobiol Dis 20: 837-848, 2005.

8. Lee KY, Bae ON, Weinstock S, Kassab M and Majid A: Neuroprotective effect of asiatic acid in rat model of focal embolic stroke. Biol Pharm Bull 37: 1397-1401, 2014.

9. Petrosillo G, Ruggiero FM, Di Venosa N and Paradies G: Decreased complex III activity in mitochondria isolated from rat heart subjected to ischemia and reperfusion: role of reactive oxygen species and cardiolipin. FASEB J 17: 714-716, 2003.

10. Liu B, Tewari AK, Zhang L, Green-Church KB, Zweier JL, Chen YR and He G: Proteomic analysis of protein tyrosine nitration after ischemia reperfusion injury: Mitochondria as the major target. Biochim Biophys Acta 1794: 476-485, 2009.

11. Peng Q, Wei $Z$ and Lau BH: Fructus corni enhances endothelial cell antioxidant defenses. Gen Pharmacol 31: 221-225, 1998.

12. Chen CC, Hsu CY, Chen CY and Liu HK: Fructus Corni suppresses hepatic gluconeogenesis related gene transcription, enhances glucose responsiveness of pancreatic beta-cells, and prevents toxin induced beta-cell death. J Ethnopharmacol 117: 483-490, 2008.

13. Wu Y, Wang X, Shen B, Kang L and Fan E: Extraction, structure and bioactivities of the polysaccharides from Fructus corni. Recent Pat Food Nutr Agric 5: 57-61, 2013.

14. Racine RJ: Modification of seizure activity by electrical stimulation. II. Motor seizure. Electroencephalogr Clin Neurophysiol 32: 281-294, 1972

15. Wang D, Ren M, Guo J, Yang G, Long X, Hu R, Shen W, Wang $X$ and Zeng K: The inhibitory effects of Npas 4 on seizures in pilocarpine-induced epileptic rats. PLoS One 9: e115801, 2014. 
16. Thummasorn S, Kumfu S, Chattipakorn S and Chattipakorn N: Granulocyte-colony stimulating factor attenuates mitochondrial dysfunction induced by oxidative stress in cardiac mitochondria. Mitochondrion 11: 457-466, 2011.

17. Qi J, Hong ZY, Xin H and Zhu YZ: Neuroprotective effects of leonurine on ischemia/reperfusion-induced mitochondrial dysfunctions in rat cerebral cortex. Biol Pharm Bull 33: 1958-1964, 2010

18. Buchan A and Pulsinelli WA: Hypothermia but not the N-methyl-D-aspartate antagonist, MK-801, attenuates neuronal damage in gerbils subjected to transient global ischemia. J Neurosci 10: 311-316, 1990.

19. Kirino T, Tamura A, Tomukai N and Sano K: Treatable ischemic neuronal damage in the gerbil hippocampus. No To Shinkei 38 : 1157-1163, 1986 (In Japanese).

20. Xie N, Li H, Wei D, LeSage G, Chen L, Wang S, Zhang Y, Chi L, Ferslew K, He L, et al: Glycogen synthase kinase- 3 and p38 MAPK are required for opioid-induced microglia apoptosis. Neuropharmacology 59: 444-451, 2010.

21. Cardenas-Rodriguez N, Huerta-Gertrudis B, Rivera-Espinosa L, Montesinos-Correa H, Bandala C, Carmona-Aparicio L and Coballase-Urrutia E: Role of oxidative stress in refractory epilepsy: Evidence in patients and experimental models. Int J Mol Sci 14: 1455-1476, 2013

22. Henshall DC: Apoptosis signalling pathways in seizure-induced neuronal death and epilepsy. Biochem Soc Trans 35: 421-423, 2007.

23. Bielli A, Scioli MG, Mazzaglia D, Doldo E and Orlandi A Antioxidants and vascular health. Life Sci 143: 209-216, 2015.

24. Vishnoi S, Raisuddin S and Parvez S: Glutamate excitotoxicity and oxidative stress in epilepsy: Modulatory role of melatonin. J Environ Pathol Toxicol Oncol 35: 365-374, 2016.

25. Zhong H, Lu J, Xia L, Zhu M and Yin H: Formation of electrophilic oxidation products from mitochondrial cardiolipin in vitro and in vivo in the context of apoptosis and atherosclerosis. Redox Biol 2: 878-883, 2014.

26. Crouser ED and Parinandi N: Mitochondrial mechanisms are at the 'heart' of novel ischemia-reperfusion therapies. Crit Care Med 39: 593-595, 2011

27. Yao K, Ye P, Zhang L, Tan J, Tang $X$ and Zhang Y: Epigallocatechin gallate protects against oxidative stress-induced mitochondria-dependent apoptosis in human lens epithelial cells. Mol Vis 14: 217-223, 2008

28. Ganie SA, Dar TA, Bhat AH, Dar KB, Anees S, Zargar MA and Masood A: Melatonin: A potential anti-oxidant therapeutic agent for mitochondrial dysfunctions and related disorders. Rejuvenation Res 19: 21-40, 2016.

29. Ralph SJ, Pritchard R, Rodríguez-Enríquez S, Moreno-Sánchez R and Ralph RK: Hitting the bull's-eye in metastatic cancers-NSAIDs elevate ROS in mitochondria, inducing malignant cell death. Pharmaceuticals (Basel) 8: 62-106, 2015.

30. Brenner D and Mak TW: Mitochondrial cell death effectors Curr Opin Cell Biol 21: 871-877, 2009.

31. Wang X: The expanding role of mitochondria in apoptosis. Genes Dev 15: 2922-2933, 2001
32. Alvarez-Jaimes L, Feliciano-Rivera M, Centeno-González M and Maldonado-Vlaar CS: Contributions of the mitogen-activated protein kinase and protein kinase $\mathrm{C}$ cascades in spatial learning and memory mediated by the nucleus accumbens. J Pharmacol Exp Ther 314: 1144-1157, 2005.

33. DeGiorgio CM, Tomiyasu U, Gott PS and Treiman DM: Hippocampal pyramidal cell loss in human status epilepticus. Epilepsia 33: 23-27, 1992.

34. Mikati MA, Abi-Habib RJ,El Sabban ME, Dbaibo GS, Kurdi RM, Kobeissi M, Farhat F and Asaad W: Hippocampal programmed cell death after status epilepticus: evidence for NMDA-receptor and ceramide-mediated mechanisms. Epilepsia 44: 282-291, 2003.

35. Spragg DD, Akar FG, Helm RH, Tunin RS, Tomaselli GF and Kass DA: Abnormal conduction and repolarization in late-activated myocardium of dyssynchronously contracting hearts. Cardiovasc Res 67: 77-86, 2005.

36. Gard JJ, Yamada K, Green KG, Eloff BC, Rosenbaum DS, Wang X, Robbins J, Schuessler RB, Yamada KA and Saffitz JE: Remodeling of gap junctions and slow conduction in a mouse model of desmin-related cardiomyopathy. Cardiovasc Res 67: 539-547, 2005.

37. Arimoto K, Fukuda H, Imajoh-Ohmi S, Saito H and Takekawa M: Formation of stress granules inhibits apoptosis by suppressing stress-responsive MAPK pathways. Nat Cell Biol 10: 1324-1332, 2008.

38. Son Y, Cheong YK, Kim NH, Chung HT, Kang DG and Pae HO: Mitogen-activated protein kinases and reactive oxygen species: How can ROS activate MAPK pathways? J Signal Transduct 2011: 792639, 2011.

39. Ledirac N, Antherieu S, D'Uby AD, Caron JC and Rahmani R: Effects of organochlorine insecticides on MAP kinase pathways in human HaCaT keratinocytes: Key role of reactive oxygen species. Toxicol Sci 86: 444-452, 2005.

40. Shao X, Luo Q, Qin Q, Qiu G and Li Z: Effect of fructus corni polysaccharides on damaged sexual function of male rats. Zhongguo Zhong Yao Za Zhi 35: 772-775, 2010 (In Chinese).

41. Yamabe N, Kang KS, Goto E, Tanaka T and Yokozawa T: Beneficial effect of Corni Fructus, a constituent of Hachimi-jio-gan, on advanced glycation end-product-mediated renal injury in Streptozotocin-treated diabetic rats. Biol Pharm Bull 30: 520-526, 2007.

42. Duan Y, Pei K, Cai H, Tu S, Zhang Z, Cheng X, Qiao F, Fan K, Qin K, Liu X and Cai B: Bioactivity evaluation-based ultra high-performance liquid chromatography coupled with electrospray ionization tandem quadrupole-time-of-flight mass spectrometry and novel distinction of multi-subchemome compatibility recognition strategy with Astragali Radix-Fructus Corni herb-pair as a case study. J Pharm Biomed Anal 129: 514-534, 2016.

43. Wang X, Liu J, Jin NA, Xu D, Wang J, Han Y and Yin N: Fructus Corni extract-induced neuritogenesis in PC12 cells is associated with the suppression of stromal interaction molecule 1 expression and inhibition of $\mathrm{Ca}^{2+}$ influx. Exp Ther Med 9: 1773-1779, 2015 . 